\title{
Iodobenzene diacetate-mediated isomerization of pyrazolyl chalcones and their cytotoxicity and anti-microbial activity
}

\author{
MAHAVIR PARSHAD ${ }^{\mathrm{a}}$, VIKAS VERMA ${ }^{\mathrm{a}}$, DEVINDER KUMAR ${ }^{\mathrm{a}, *}$, \\ BALASUBRAMANIAN NARASIMHAN ${ }^{\mathrm{b}}$, SMIT KOUR $^{\mathrm{c}}$, SHASHANK SINGH ${ }^{\mathrm{c}}$ and \\ PAYARE LAL SANGWAN ${ }^{\mathrm{C}}$

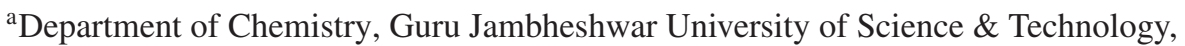 \\ Hisar 125001, Haryana, India \\ ${ }^{b}$ Faculty of Pharmaceutical Sciences, M D University, Rohtak 124 001, Haryana, India \\ ${ }^{\mathrm{c}}$ Cancer Pharmacology Division and Bioorganic Chemistry Division, CSIR-Indian \\ Institute of Integrative Medicine, Jammu 180 001, India \\ e-mail: dk_ic@yahoo.com
}

MS received 8 March 2014; revised 31 August 2014; accepted 16 September 2014

\begin{abstract}
Synthesis of cis (E)-1-(5-hydroxy-3-methyl-1-phenyl-1 $H$-pyrazol-4-yl)-3-phenyl/aryl/heteroarylprop-2-en-1-ones from 1-phenyl-3-methyl-4-acetylpyrazol-5-one was achieved in good yield. s-cis (E)-1-(5Hydroxy-3-methyl-1-phenyl-1 $H$-pyrazol-4-yl)-3-arylprop-2-en-1-ones were isomerized to $s$-trans $(E)-4$-(3(phenyl/aryl/heteroaryl)acryloyl)-5-methyl-2-phenyl-1 $H$-pyrazol-3 $(2 H)$-ones using iodobenzene diacetate in dichloromethane at room temperature in excellent yield. The structure and geometry of these $\alpha, \beta$-unsaturated ketones (pyrazolyl ketones) were established with the help of NMR, 2D NMR and HRMS techniques. The cytotoxicity of pyrazolyl chalcones showed that s-cis (E) 1-(5-Hydroxy-3-methyl-1-phenyl-1H-pyrazol-4-yl)3-(4-methylphenyl)-prop-2-en-1-one is active at very low concentrations $\left(\mathrm{IC}_{50} 13.3 \mu \mathrm{M}\right)$ against colon cancer cell line (HCT-116). The in vitro anti-microbial studies of pyrazolyl chalcones were also tested against grampositive (B. subtilis, S. aureus) and gram-negative bacteria (E. coli) and for anti-fungal activity against $C$. albicans and A. niger.
\end{abstract}

Keywords. Pyrazolyl chalcone; iodobenzene diacetate; cytotoxicity; anti-microbial.

\section{Introduction}

The $\alpha, \beta$-unsaturated ketones (chalcones) are open chain compounds consisting of two aromatic rings joined by a three carbon enone system and constitute an attractive molecular scaffolds for the search of new biologically active flavonoids and isoflavonoids. ${ }^{1-4}$ The incorporation of pyrazole moiety in chalcones has been considered of great interest due to their better biological activities i.e., anti-microbial, ${ }^{5}$ anti-infective, ${ }^{6}$ cytotoxic, ${ }^{7}$ anti-invasive, ${ }^{8}$ anti-oxidant and anti-inflammatory activity. ${ }^{9}$ Further, it has been reported that the presence of enone functionality in chalcones having pyrazole moiety enhance their biological activity. ${ }^{10}$ Recently, there has been much interest in the utilisation of hypervalent iodine reagents for many useful organic transformations and synthesis of important active intermediates and biologically active molecules. ${ }^{11}$

\footnotetext{
${ }^{*}$ For correspondence
}

Prompted by the above observations and our own interest in this field, ${ }^{12}$ we report herein an improved synthesis of cis (E)-1-(5-hydroxy-3-methyl-1-phenyl-1 $H$ pyrazol-4-yl)-3-phenyl/aryl/heteroarylprop-2-en-1-ones (pyrazolyl chalcones, 2) by condensation of 1-phenyl-3methyl-4-acetylpyrazol-5-one (1) and aromatic aldehydes in good yield. Further, the pyrazolyl chalcones (2) are isomerized to $s$-trans $(E)$-4-(3-(phenyl/aryl/hetroaryl) acryloyl)-5-methyl-2-phenyl-1H-pyrazol-3(2H)-ones (3) using iodobenzene diacetate (commonly used hypervalent iodine reagent) in dichloromethane by stirring at room temperature in excellent yield (90-98\%) within 0.5 to $1 \mathrm{~h}$. To the best of our knowledge the $s$-cis to $s$-trans isomerization is probably the first report of its kind. The cytotoxicity of these pyrazolyl chalcones ( 2 and $\mathbf{3}$ ) is studied against five cancer cell lines. These compounds were also tested in vitro for their antibacterial activity against gram-positive (B. subtilis, $S$. aureus) and gram-negative bacteria (E. coli) and for anti-fungal activity against $C$. albicans and $A$. niger as well. 


\section{Experimental}

\subsection{General}

Melting points were determined in open capillaries and are uncorrected. Iodobenzene diacetate was purchased from Aldrich. FTIR spectra were obtained in $\mathrm{KBr}$ on IR Affinity-I (Shimadzu) spectrophotometer and are reported in $\mathrm{cm}^{-1} \cdot{ }^{1} \mathrm{H},{ }^{13} \mathrm{C}$ NMR, DEPT-135, 1D NOE difference spectra and 2D-NMR, COSY (correlation spectroscopy), HSQC (heteronuclear single-quantum coherence), HMBC (heteronuclear multiple bond correlation), TOCSY (total correlation spectroscopy) and ROESY (rotating frame enhancement spectroscopy) spectra were scanned on a Bruker Avance III NMR spectrometer operating at $400 \mathrm{MHz}$ in $\mathrm{CDCl}_{3}$ and are expressed as ppm with respect to TMS. HRMS were recorded on the 6500 series Agilent Accurate-Mass Q-TOF LC/MS system.

2.2 General procedure for the Synthesis of s-cis (E)-1(5-hydroxy-3-methyl-1-phenyl-1H-pyrazol-4-yl)-3phenyl/aryl/heteroarylprop-2-en-1-ones (2)

1-Phenyl-3-methylpyrazol-5-one $(4.002 \mathrm{~g}, 0.023 \mathrm{~mol})$ was dissolved by heating in sodium dried dioxane $(150 \mathrm{~mL})$ in a $250 \mathrm{~mL}$ round bottom flask. The solution was cooled to room temperature and calcium hydroxide $(3.33 \mathrm{~g}, 0.045 \mathrm{~mol})$ was added in portions followed by acetyl chloride $(2.19 \mathrm{~g}, 0.028 \mathrm{~mol})$. The reaction mixture was refluxed for $30 \mathrm{~min}$ followed by removal of excess dioxane under vacuum. The residue was treated with $10 \% \mathrm{HCl}$ and the resulting mixture was extracted with dichloromethane $(2 \times 30 \mathrm{~mL})$. The excess dichloromethane was distilled off to give the solid which was recrystalized with aqueous methanol to afford 1-phenyl-3-methyl-4-acetylpyrazol-5-one (1). Reaction of 1 with various substituted aromatic aldehydes (1:1 molar ratio) in chloroform using piperidine in catalytic amount under reflux for 5-6 h furnished $c$ is (E)-1-(5-hydroxy-3-methyl-1-phenyl-1H-pyrazol-4-yl)3-phenyl/aryl/heteroarylprop-2-en-1-ones (2) in good yield.

2.2a s-cis (E) 1-(5-Hydroxy-3-methyl-1-phenyl-1Hpyrazol-4-yl)-3-phenyl-prop-2-en-1-one (2a): M.p.: $158-159^{\circ} \mathrm{C}$ (lit. ${ }^{13}$ M.p. $158^{\circ} \mathrm{C}$ ); yield: $84 \%$; $\operatorname{IR}(\mathrm{KBr}) v$ : $1625,1564,1517,1427 \mathrm{~cm}^{-1}$; NMR $\delta_{\mathrm{H}}(400 \mathrm{MHz}$, $\left.\mathrm{CDCl}_{3}\right)$ : 7.96-7.91 (m, 2H, $\left.\mathrm{C}_{2^{\prime}}-\mathrm{H}, \mathrm{C}_{6^{\prime}}-\mathrm{H}\right), 7.94(\mathrm{~d}$, $\left.1 \mathrm{H}, J=15.6 \mathrm{~Hz}, \mathrm{C}_{8}-\mathrm{H}\right), 7.64-7.62\left(\mathrm{~m}, 2 \mathrm{H}, \mathrm{C}_{2^{\prime \prime}}-\mathrm{H}\right.$, $\left.\mathrm{C}_{6 \prime \prime}-\mathrm{H}\right), 7.46-7.42\left(\mathrm{~m}, 5 \mathrm{H}, \mathrm{C}_{3^{\prime}}-\mathrm{H}, \mathrm{C}_{5^{\prime}}-\mathrm{H}, \mathrm{C}_{3^{\prime \prime}}-\mathrm{H}, \mathrm{C}_{5^{\prime \prime}}-\mathrm{H}\right.$, $\left.\mathrm{C}_{4^{\prime \prime}}-\mathrm{H}\right), 7.26-7.23\left(\mathrm{~m}, 1 \mathrm{H}, \mathrm{C}_{4^{\prime}}-\mathrm{H}\right), 7.16(\mathrm{~d}, 1 \mathrm{H}, J=$ $\left.15.6 \mathrm{~Hz}, \mathrm{C}_{7}-\mathrm{H}\right), 2.59\left(\mathrm{~s}, 3 \mathrm{H}, \mathrm{C}_{3}-\mathrm{CH}_{3}\right)$; NMR $\delta_{\mathrm{C}}(100$
$\left.\mathrm{MHz}, \mathrm{CDCl}_{3}\right): 178.0$ (C-6), 165.2 (C-5), 147.0 (C-3), 144.1 (C-8), $137.7\left(\mathrm{C}-1^{\prime}\right), 134.4\left(\mathrm{C}-1^{\prime \prime}\right), 131.1\left(\mathrm{C}-4^{\prime}\right)$, $129.2\left(\mathrm{C}-2^{\prime \prime} / \mathrm{C}-6^{\prime \prime}\right), 129.1\left(\mathrm{C}-3^{\prime \prime} / \mathrm{C}-5^{\prime \prime}\right), 129.0,128.6(\mathrm{C}-$ 3'/C-5'), 125.9 (C-4"), 119.8 (C-2'/C-6'), 119.2 (C-7), 104.7 (C-4), $16.5\left(\mathrm{C}_{3}-\mathrm{CH}_{3}\right)$; HRMS: $\mathrm{m} / \mathrm{z}\left(\mathrm{M}^{+}\right)$calcd. for $\mathrm{C}_{19} \mathrm{H}_{16} \mathrm{~N}_{2} \mathrm{O}_{2}: 304.1206$, found: $305.1278(\mathrm{M}+\mathrm{H})$.

2.2b s-cis (E) 1-(5-Hydroxy-3-methyl-1-phenyl-1Hpyrazol-4-yl)-3-(4-methoxyphenyl)-prop-2-en-1-one (2b): M.p.: $152-153^{\circ} \mathrm{C}$ (lit. ${ }^{13}$ M.p. $153^{\circ} \mathrm{C}$ ); yield: $88 \%$; IR $(\mathrm{KBr}) v: 3169,1631,1568,1516 \mathrm{~cm}^{-1} ; \mathrm{NMR} \delta_{\mathrm{H}}(400$ $\mathrm{MHz}, \mathrm{CDCl}_{3}$ ): 7.94-7.92 (m, 2H, $\left.\mathrm{C}_{2^{\prime}}-\mathrm{H}, \mathrm{C}_{6^{\prime}}-\mathrm{H}\right), 7.91$ $\left(\mathrm{d}, 1 \mathrm{H}, J=15.5 \mathrm{~Hz}, \mathrm{C}_{8}-\mathrm{H}\right), 7.59(\mathrm{~d}, 2 \mathrm{H}, J=8.7 \mathrm{~Hz}$, $\left.\mathrm{C}_{2^{\prime \prime}}-\mathrm{H}, \mathrm{C}_{6^{\prime \prime}}-\mathrm{H}\right), 7.44\left(\mathrm{t}, 2 \mathrm{H}, J=7.6,6.5 \mathrm{~Hz}, \mathrm{C}_{3^{\prime}}-\mathrm{H}, \mathrm{C}_{5^{\prime}}\right.$ $\mathrm{H}), 7.26-7.22\left(\mathrm{~m}, 1 \mathrm{H}, \mathrm{C}_{4^{\prime}}-\mathrm{H}\right), 7.01(\mathrm{~d}, 1 \mathrm{H}, J=15.5$ $\left.\mathrm{Hz}, \mathrm{C}_{7}-\mathrm{H}\right), 6.96\left(\mathrm{~d}, 2 \mathrm{H}, J=8.7 \mathrm{~Hz}, \mathrm{C}_{3^{\prime \prime}}-\mathrm{H}, \mathrm{C}_{5^{\prime \prime}}-\mathrm{H}\right)$, $3.87\left(\mathrm{~s}, 3 \mathrm{H}, \mathrm{C}_{4^{\prime \prime}}-\mathrm{OCH}_{3}\right), 2.58\left(\mathrm{~s}, 3 \mathrm{H}, \mathrm{C}_{3}-\mathrm{CH}_{3}\right)$; NMR $\delta_{\mathrm{C}}\left(100 \mathrm{MHz}, \mathrm{CDCl}_{3}\right): 177.6(\mathrm{C}-6), 165.6(\mathrm{C}-5), 162.2$ (C-4"), 147.0 (C-3), 144.0 (C-8), 137.8 (C-1'), 130.5 (C-2"/C-6"), $129.0\left(\mathrm{C}-3^{\prime} / \mathrm{C}-5^{\prime}\right), 127.2\left(\mathrm{C}-1^{\prime \prime}\right), 125.7$ (C$\left.4^{\prime}\right), 119.7\left(\mathrm{C}-2^{\prime} / \mathrm{C}-6^{\prime}\right), 116.4(\mathrm{C}-7), 114.6\left(\mathrm{C}-3^{\prime \prime} / \mathrm{C}-5^{\prime \prime}\right)$, $104.3(\mathrm{C}-4), 55.5\left(\mathrm{C}_{4^{\prime \prime}}-\mathrm{OCH}_{3}\right), 16.5\left(\mathrm{C}_{3}-\mathrm{CH}_{3}\right)$; HRMS: $\mathrm{m} / \mathrm{z}\left(\mathrm{M}^{+}\right)$calcd. for $\mathrm{C}_{20} \mathrm{H}_{18} \mathrm{~N}_{2} \mathrm{O}_{3}: 334.1314$, found: $335.1386(\mathrm{M}+\mathrm{H})$.

2.2c s-cis (E) 1-(5-Hydroxy-3-methyl-1-phenyl-1Hpyrazol-4-yl)-3-(4-methylphenyl)-prop-2-en-1-one (2c): M.p.: $158-159^{\circ} \mathrm{C}$ (lit. ${ }^{13}$ M.p. $158^{\circ} \mathrm{C}$ ); yield: $87 \%$; $\operatorname{IR}(\mathrm{KBr}) v: 1600,1597,1543 \mathrm{~cm}^{-1}$; NMR $\delta_{\mathrm{H}}(400 \mathrm{MHz}$, $\left.\mathrm{CDCl}_{3}\right)$ : 7.95-7.89 (m, 2H, C $\left.2^{\prime}-\mathrm{H}, \mathrm{C}_{6^{\prime}}-\mathrm{H}\right), 7.88(\mathrm{~d}, 1 \mathrm{H}$, $\left.J=14.8 \mathrm{~Hz}, \mathrm{C}_{8}-\mathrm{H}\right), 7.53\left(\mathrm{~d}, 2 \mathrm{H}, J=7.6 \mathrm{~Hz}, \mathrm{C}_{2^{\prime \prime}}-\right.$ $\left.\mathrm{H}, \mathrm{C}_{6^{\prime \prime}}-\mathrm{H}\right), 7.44-7.40\left(\mathrm{~m}, 2 \mathrm{H}, \mathrm{C}_{3^{\prime}}-\mathrm{H}, \mathrm{C}_{5^{\prime}}-\mathrm{H}\right), 7.29-7.21$ $\left(\mathrm{m}, 3 \mathrm{H}, \mathrm{C}_{3^{\prime \prime}}-\mathrm{H}, \mathrm{C}_{5^{\prime \prime}}-\mathrm{H}, \mathrm{C}_{4^{\prime}}-\mathrm{H}\right), 7.11(\mathrm{~d}, 1 \mathrm{H}, J=14.8$ $\left.\mathrm{Hz}, \mathrm{C}_{7}-\mathrm{H}\right), 2.61\left(\mathrm{~s}, 3 \mathrm{H}, \mathrm{C}_{3}-\mathrm{CH}_{3}\right), 2.42\left(\mathrm{~s}, 3 \mathrm{H}, \mathrm{C}_{4^{\prime \prime}}-\mathrm{CH}_{3}\right)$; NMR $\delta_{\mathrm{C}}\left(100 \mathrm{MHz}, \mathrm{CDCl}_{3}\right): 177.8(\mathrm{C}-6), 165.5(\mathrm{C}-$ 5), 147.0 (C-3), 144.2 (C-8), 141.9 (C-4"), 137.7 (C$\left.1^{\prime}\right), 131.7\left(\mathrm{C}-1^{\prime \prime}\right), 129.9$ (C-2"/C-6"), $129.0\left(\mathrm{C}-3^{\prime \prime} / \mathrm{C}-5^{\prime \prime}\right)$, $128.6\left(\mathrm{C}-3^{\prime} / \mathrm{C}-5^{\prime}\right), 125.8\left(\mathrm{C}-4^{\prime}\right), 119.8\left(\mathrm{C}-2^{\prime} / \mathrm{C}-6^{\prime}\right), 118.0$ (C-7), 104.4 (C-4), $21.6\left(\mathrm{C}_{4^{\prime \prime}}-\mathrm{CH}_{3}\right), 15.4\left(\mathrm{C}_{3}-\mathrm{CH}_{3}\right)$; HRMS: $\mathrm{m} / \mathrm{z}\left(\mathrm{M}^{+}\right)$calcd. for $\mathrm{C}_{20} \mathrm{H}_{18} \mathrm{~N}_{2} \mathrm{O}_{2}: 318.1367$, found: $319.1440(\mathrm{M}+\mathrm{H})$.

2.2d s-cis (E) 1-(5-Hydroxy-3-methyl-1-phenyl-1Hpyrazol-4-yl)-3-(4-chlorophenyl)-prop-2-en-1-one (2d): M.p.: $171-172{ }^{\circ} \mathrm{C}$; yield: $83 \%$; $\operatorname{IR}(\mathrm{KBr}) v$ : 1788,1620 , $1579,1570,1560 \mathrm{~cm}^{-1} ; \mathrm{NMR} \delta_{\mathrm{H}}\left(400 \mathrm{MHz}, \mathrm{CDCl}_{3}\right)$ : $7.90\left(\mathrm{~d}, 1 \mathrm{H}, J=7.6 \mathrm{~Hz}, \mathrm{C}_{2^{\prime}}-\mathrm{H}, \mathrm{C}_{6^{\prime}}-\mathrm{H}\right), 7.87(\mathrm{~d}, 1 \mathrm{H}$, $\left.J=15.6 \mathrm{~Hz}, \mathrm{C}_{8}-\mathrm{H}\right), 7.55\left(\mathrm{~d}, 2 \mathrm{H}, J=8.4 \mathrm{~Hz}, \mathrm{C}_{3^{\prime \prime}}-\mathrm{H}\right.$, $\left.\mathrm{C}_{5^{\prime \prime}}-\mathrm{H}\right), 7.46-7.41\left(\mathrm{~m}, 4 \mathrm{H}, \mathrm{C}_{3^{\prime}}-\mathrm{H}, \mathrm{C}_{5^{\prime}}-\mathrm{H}, \mathrm{C}_{2^{\prime \prime}}-\mathrm{H}, \mathrm{C}_{6^{\prime \prime}}-\mathrm{H}\right)$, $7.26\left(\mathrm{~m}, 1 \mathrm{H}, \mathrm{C}_{4^{\prime}}-\mathrm{H}\right), 7.15\left(\mathrm{~d}, 1 \mathrm{H}, J=15.6 \mathrm{~Hz}, \mathrm{C}_{7^{-}}\right.$ $\mathrm{H}), 2.58\left(\mathrm{~s}, 3 \mathrm{H}, \mathrm{C}_{3}-\mathrm{CH}_{3}\right)$; $\mathrm{NMR} \delta_{\mathrm{C}}\left(100 \mathrm{MHz}, \mathrm{CDCl}_{3}\right)$ : 177.9 (C-6), 165.0 (C-5), 146.9 (C-3), 142.5 (C-8), 
$137.6\left(\mathrm{C}-1^{\prime}\right), 137.0\left(\mathrm{C}-1^{\prime \prime}\right), 132.9\left(\mathrm{C}-2^{\prime \prime} / \mathrm{C}-6^{\prime \prime}\right), 129.7$ (C-3"/C-5"), 129.4 (C-3'/C-5'), 129.0 (C-4'), 126.0 (C4"), 119.9 (C-2'/C-6'), 119.8 (C-7), 104.7 (C-4), 16.5 $\left(\mathrm{C}_{3}-\mathrm{CH}_{3}\right)$; HRMS: $\mathrm{m} / \mathrm{z}\left(\mathrm{M}^{+}\right)$calcd. for $\mathrm{C}_{19} \mathrm{H}_{15} \mathrm{ClN}_{2} \mathrm{O}_{2}$ : 338.0826, found: $339.0899(\mathrm{M}+\mathrm{H})$.

2.2e s-cis (E) 1-(5-Hydroxy-3-methyl-1-phenyl-1Hpyrazol-4-yl)-3-(2-furyl)-prop-2-en-1-one (2e): M.p.: $168-169^{\circ} \mathrm{C}$ (lit. ${ }^{13}$ M.p. $168^{\circ} \mathrm{C}$ ); yield: $82 \%$; $\operatorname{IR}(\mathrm{KBr}) v$ : 3093, 1734, 1604, $1521 \mathrm{~cm}^{-1}$; NMR $\delta_{\mathrm{H}}(400 \mathrm{MHz}$, $\left.\mathrm{CDCl}_{3}\right): 7.92\left(\mathrm{~d}, 2 \mathrm{H}, J=8.0 \mathrm{~Hz}, \mathrm{C}_{2^{\prime}}-\mathrm{H}, \mathrm{C}_{6^{\prime}}-\mathrm{H}\right), 7.66$ $\left(\mathrm{d}, 1 \mathrm{H}, J=15.2 \mathrm{~Hz}, \mathrm{C}_{8}-\mathrm{H}\right), 7.58\left(\mathrm{~s}, 1 \mathrm{H}, \mathrm{C}_{5^{\prime \prime}}-\mathrm{H}\right), 7.43(\mathrm{t}$, $\left.2 \mathrm{H}, J=8.0,7.6 \mathrm{~Hz}, \mathrm{C}_{3^{\prime}}-\mathrm{H}, \mathrm{C}_{5^{\prime}}-\mathrm{H}\right), 7.23(\mathrm{t}, 1 \mathrm{H}, J=7.2$ $\left.\mathrm{Hz}, \mathrm{C}_{4^{\prime}}-\mathrm{H}\right), 7.01\left(\mathrm{~d}, 1 \mathrm{H}, J=15.2 \mathrm{~Hz}, \mathrm{C}_{7}-\mathrm{H}\right), 6.76$ (br s, $\left.1 \mathrm{H}, \mathrm{C}_{3^{\prime \prime}}-\mathrm{H}\right), 6.55$ (br s, $\left.1 \mathrm{H}, \mathrm{C}_{4^{\prime \prime}}-\mathrm{H}\right), 2.56\left(\mathrm{~s}, 3 \mathrm{H}, \mathrm{C}_{3^{-}}\right.$ $\left.\mathrm{CH}_{3}\right)$; NMR $\delta_{\mathrm{C}}\left(100 \mathrm{MHz}, \mathrm{CDCl}_{3}\right): 177.0(\mathrm{C}-6), 165.5$ (C-5), $158.9\left(\mathrm{C}-2^{\prime \prime}\right), 151.3(\mathrm{C}-3), 145.8$ (C-8), 137.8 (C$\left.1^{\prime}\right), 129.7\left(\mathrm{C}-5^{\prime \prime}\right), 129.0\left(\mathrm{C}-3^{\prime} / \mathrm{C}-5^{\prime}\right), 125.7\left(\mathrm{C}-4^{\prime}\right), 119.7$ (C-2'/C-6'), 117.3 (C-7), 116.5 (C-3"), 113.0 (C-4"), 104.8 (C-4) $16.4\left(\mathrm{C}_{3}-\mathrm{CH}_{3}\right)$; HRMS: m/z $\left(\mathrm{M}^{+}\right)$calcd. for $\mathrm{C}_{17} \mathrm{H}_{14} \mathrm{~N}_{2} \mathrm{O}_{3}$ : 294.1009, found: $295.1076(\mathrm{M}+\mathrm{H})$.

$2.2 \mathrm{f} s$-cis (E) 1-(5-Hydroxy-3-methyl-1-phenyl-1Hpyrazol-4-yl)-3-(4-bromophenyl)-prop-2-en-1-one (2f): M.p.: $169-170^{\circ} \mathrm{C}$; yield: $85 \%$; IR(KBr)v: 3059, 2927 , 1631, 1573, 1504, $1419 \mathrm{~cm}^{-1}$; NMR $\delta_{\mathrm{H}}(400 \mathrm{MHz}$, $\mathrm{CDCl}_{3}$ ):); 7.91 (d, 2H, $\left.J=7.6 \mathrm{~Hz}, \mathrm{C}_{2^{\prime}}-\mathrm{H}, \mathrm{C}_{6^{\prime}}-\mathrm{H}\right), 7.85$ $\left(\mathrm{d}, 1 \mathrm{H}, J=15.6 \mathrm{~Hz}, \mathrm{C}_{8}-\mathrm{H}\right), 7.57(\mathrm{~d}, 2 \mathrm{H}, J=8.4 \mathrm{~Hz}$, $\left.\mathrm{C}_{3^{\prime \prime}}-\mathrm{H}, \mathrm{C}_{5^{\prime \prime}}-\mathrm{H}\right), 7.46\left(\mathrm{~d}, 2 \mathrm{H}, J=8.4 \mathrm{~Hz}, \mathrm{C}_{2^{\prime \prime}}-\mathrm{H}, \mathrm{C}_{6^{\prime \prime}}-\mathrm{H}\right)$, $7.42\left(\mathrm{~d}, 2 \mathrm{H}, J=8.4 \mathrm{~Hz}, \mathrm{C}_{3^{\prime}}-\mathrm{H}, \mathrm{C}_{5^{\prime}}-\mathrm{H}\right), 7.24(\mathrm{~d}, 1 \mathrm{H}$, $\left.J=7.4 \mathrm{~Hz}, \mathrm{C}_{4^{\prime}}-\mathrm{H}\right), 7.13\left(\mathrm{~d}, 1 \mathrm{H}, J=15.6 \mathrm{~Hz}, \mathrm{C}_{7}-\mathrm{H}\right)$, $2.58\left(\mathrm{~s}, 3 \mathrm{H}, \mathrm{C}_{3}-\mathrm{CH}_{3}\right) ; \mathrm{NMR} \delta_{\mathrm{C}}\left(100 \mathrm{MHz}, \mathrm{CDCl}_{3}\right)$ : 177.9 (C-6), 165.0 (C-5), 146.9 (C-3), 142.6 (C-8), $137.6\left(\mathrm{C}-1^{\prime}\right), 133.3\left(\mathrm{C}-1^{\prime \prime}\right), 132.4\left(\mathrm{C}-2^{\prime \prime} / \mathrm{C}-6^{\prime \prime}\right), 129.8$ (C-3"/C-5"), 129.0 (C-3'/C-5'), 126.0 (C-4'), 125.4 (C-4"), 119.9 (C-2'/C-6'), 119.8 (C-7), 104.8 (C-4), $16.5\left(\mathrm{C}_{3}-\mathrm{CH}_{3}\right)$; HRMS: $\mathrm{m} / \mathrm{z}\left(\mathrm{M}^{+}\right)$calcd. for $\mathrm{C}_{19} \mathrm{H}_{15}$ $\mathrm{BrN}_{2} \mathrm{O}_{2}: 382.0315$, found: $383.0389(\mathrm{M}+\mathrm{H})$.

2.3 General procedure for the Synthesis of s-trans (E)-4-(3-(phenyl/aryl/hetroaryl)acryloyl)-5-methyl-2phenyl-1H-pyrazol-3(2H)-ones (3)

To the solution of cis (E)-1-(5-hydroxy-3-methyl-1phenyl-1 $H$-pyrazol-4-yl)-3-phenyl/aryl/heteroarylprop2-en-1-ones (2) $(0.001 \mathrm{~mol})$ in dichloromethane $15 \mathrm{~mL}$, iodobenzene diacetate $(0.001 \mathrm{~mol})$ was added in small portions in $10 \mathrm{~min}$. The reaction mixture was further stirred for 0.5 to $1 \mathrm{~h}$ at room temperature till the completion of reaction as monitored by TLC. The excess dichloromethane was distilled off to give a solid mass which was triturated with hexane to remove the iodosobenzene followed by recrystalization with dichloromethane and hexane to obtain s-trans $(E)-4$ (3-(phenyl/aryl/hetroaryl)acryloyl)-5-methyl-2-phenyl$1 H$-pyrazol-3(2H)-ones (3).

2.3a s-trans (E) 4-Cinnamoyl-5-methyl-2-phenyl-1Hpyrazol-3(2H)-one (3a): M.p.: $142-143^{\circ} \mathrm{C}$; yield: $90 \%$; IR(KBr) v: $3371,1712,1597 \mathrm{~cm}^{-1} ; \mathrm{NMR} \delta_{\mathrm{H}}(400$ $\left.\mathrm{MHz}, \mathrm{CDCl}_{3}\right): 7.92\left(\mathrm{~d}, 1 \mathrm{H}, J=15.3 \mathrm{~Hz}, \mathrm{C}_{8}-\mathrm{H}\right), 7.82-$ $7.77\left(\mathrm{~m}, 2 \mathrm{H}, \mathrm{C}_{2^{\prime}}-\mathrm{H}, \mathrm{C}_{6^{\prime}}-\mathrm{H}\right), 7.51-7.16\left(\mathrm{~m}, 8 \mathrm{H}, \mathrm{C}_{3^{\prime \prime}}-\mathrm{H}\right.$, $\left.\mathrm{C}_{5^{\prime \prime}}-\mathrm{H}, \mathrm{C}_{2^{\prime \prime}}-\mathrm{H}, \mathrm{C}_{6^{\prime \prime}}-\mathrm{H}, \mathrm{C}_{3^{\prime}}-\mathrm{H}, \mathrm{C}_{5^{\prime}}-\mathrm{H}, \mathrm{C}_{4^{\prime}}-\mathrm{H}, \mathrm{C}_{4^{\prime \prime}}-\mathrm{H}\right), 6.65$ $\left(\mathrm{d}, 1 \mathrm{H}, J=15.3 \mathrm{~Hz}, \mathrm{C}_{7}-\mathrm{H}\right), 2.59\left(\mathrm{~s}, 3 \mathrm{H}, \mathrm{C}_{3}-\mathrm{CH}_{3}\right), 1.55$ (br s, $1 \mathrm{H}, \mathrm{NH})$; NMR $\delta_{\mathrm{C}}\left(100 \mathrm{MHz}, \mathrm{CDCl}_{3}\right): 183.4(\mathrm{C}-$ 6), 165.4 (C-5), 158.9 (C-3), 148.1 (C-8), $137.1\left(\mathrm{C}-1^{\prime}\right)$, $133.6\left(\mathrm{C}-1^{\prime \prime}\right), 131.6\left(\mathrm{C}-4^{\prime \prime}\right), 129.1\left(\mathrm{C}-3^{\prime \prime} / \mathrm{C}-5^{\prime \prime}\right), 129.0$ $\left(\mathrm{C}-2^{\prime \prime} / \mathrm{C}-6^{\prime \prime}\right), 128.9\left(\mathrm{C}-3^{\prime} / \mathrm{C}-5^{\prime}\right) 126.0\left(\mathrm{C}-4^{\prime}\right), 119.4$ (C2'/C-6'), 118.6 (C-7), $20.8\left(\mathrm{C}_{3}-\mathrm{CH}_{3}\right)$; HRMS: m/z ( $\left.\mathrm{M}^{+}\right)$ calcd. for $\mathrm{C}_{19} \mathrm{H}_{16} \mathrm{~N}_{2} \mathrm{O}_{2}$ : 304.1205, found: 305.1278 $(\mathrm{M}+\mathrm{H})$.

2.3b s-trans (E) 4-(3-(4-Methoxyphenyl)acryloyl)5-methyl-2-phenyl-1H-pyrazol-3(2H)-one (3b): M.p.: 140-141 ${ }^{\circ} \mathrm{C}$; yield: $94 \%$; IR(KBr)v: 3157, 1705, 1581, $1506 \mathrm{~cm}^{-1} ; \mathrm{NMR} \delta_{\mathrm{H}}\left(400 \mathrm{MHz}, \mathrm{CDCl}_{3}\right) ; 7.87(\mathrm{~d}, 1 \mathrm{H}$, $\left.J=15.2 \mathrm{~Hz}, \mathrm{C}_{8}-\mathrm{H}\right), 7.81\left(\mathrm{~d}, 2 \mathrm{H}, J=7.9 \mathrm{~Hz}, \mathrm{C}_{2^{\prime}}-\mathrm{H}\right.$, $\left.\mathrm{C}_{6^{\prime}}-\mathrm{H}\right), 7.46\left(\mathrm{~d}, 2 \mathrm{H}, J=8.6 \mathrm{~Hz}, \mathrm{C}_{2^{\prime \prime}}-\mathrm{H}, \mathrm{C}_{6^{\prime \prime}}-\mathrm{H}\right), 7.34(\mathrm{t}$, $\left.2 \mathrm{H}, J=8.0,7.8 \mathrm{~Hz}, \mathrm{C}_{3^{\prime}}-\mathrm{H}, \mathrm{C}_{5^{\prime}}-\mathrm{H}\right), 7.17(\mathrm{t}, \mathrm{IH}, J=7.3$ $\left.\mathrm{Hz}, \mathrm{C}_{4^{\prime}}-\mathrm{H}\right), 6.87\left(\mathrm{~d}, 2 \mathrm{H}, J=8.6 \mathrm{~Hz}, \mathrm{C}_{3^{\prime}}-\mathrm{H}, \mathrm{C}_{5^{\prime \prime}}-\mathrm{H}\right), 6.52$ $\left(\mathrm{d}, 1 \mathrm{H}, J=15.2 \mathrm{~Hz}, \mathrm{C}_{7}-\mathrm{H}\right), 3.82\left(\mathrm{~s}, 3 \mathrm{H}, \mathrm{C}_{4^{\prime \prime}}-\mathrm{OCH}_{3}\right)$, $2.62\left(\mathrm{~s}, 3 \mathrm{H}, \mathrm{C}_{3}-\mathrm{CH}_{3}\right), 1.55$ (br s, $\left.1 \mathrm{H}, \mathrm{NH}\right)$; NMR $\delta_{\mathrm{C}}$ $\left(100 \mathrm{MHz}, \mathrm{CDCl}_{3}\right): 183.3$ (C-6), 165.7 (C-5), 162.5 (C$\left.4^{\prime \prime}\right), 159.2$ (C-3), 147.8 (C-8), $137.2\left(\mathrm{C}-1^{\prime}\right), 131.0$ (C$\left.2^{\prime \prime} / \mathrm{C}-6^{\prime \prime}\right), 128.9\left(\mathrm{C}-3^{\prime} / \mathrm{C}-5^{\prime}\right), 126.4\left(\mathrm{C}-1^{\prime \prime}\right), 125.9\left(\mathrm{C}-4^{\prime}\right)$, $119.4\left(\mathrm{C}-2^{\prime} / \mathrm{C}-6^{\prime}\right), 116.1$ (C-7), $114.5\left(\mathrm{C}-3^{\prime \prime} / \mathrm{C}-5^{\prime \prime}\right), 54.4$ $\left(\mathrm{C}_{4^{\prime \prime}}-\mathrm{OCH}_{3}\right), 20.8\left(\mathrm{C}_{3}-\mathrm{CH}_{3}\right)$; HRMS: $\mathrm{m} / \mathrm{z}\left(\mathrm{M}^{+}\right)$calcd. for $\mathrm{C}_{20} \mathrm{H}_{18} \mathrm{~N}_{2} \mathrm{O}_{3}$ : 334.1315, found: $335.1386(\mathrm{M}+\mathrm{H})$.

2.3c s-trans (E) 5-Methyl-2-phenyl-4-(3-p-tolylacryloyl)1H-pyrazol-3(2H)-one (3c): M.p.: $144-145^{\circ} \mathrm{C}$; yield: 90\%; IR(KBr)v: $3367,1710,1589 \mathrm{~cm}^{-1} ; \mathrm{NMR} \delta_{\mathrm{H}}(400$ $\left.\mathrm{MHz}, \mathrm{CDCl}_{3}\right): 7.89\left(\mathrm{~d}, 1 \mathrm{H}, J=15.2 \mathrm{~Hz}, \mathrm{C}_{8}-\mathrm{H}\right), 7.81$ $\left(\mathrm{d}, 2 \mathrm{H}, J=8.0 \mathrm{~Hz}, \mathrm{C}_{2^{\prime}}-\mathrm{H}, \mathrm{C}_{6^{\prime}}-\mathrm{H}\right), 7.39(\mathrm{~d}, 2 \mathrm{H}, J=$ $\left.8.0 \mathrm{~Hz}, \mathrm{C}_{2^{\prime \prime}}-\mathrm{H}, \mathrm{C}_{6^{\prime \prime}}-\mathrm{H}\right), 7.35\left(\mathrm{t}, 2 \mathrm{H}, \mathrm{J}=8.0 \mathrm{~Hz}, \mathrm{C}_{3^{\prime}}-\mathrm{H}\right.$, $\left.\mathrm{C}_{5^{\prime}}-\mathrm{H}\right), 7.20-7.16\left(\mathrm{~m}, 3 \mathrm{H}, \mathrm{C}_{3^{\prime \prime}}-\mathrm{H}, \mathrm{C}_{5^{\prime \prime}}-\mathrm{H}, \mathrm{C}_{4^{\prime}}-\mathrm{H}\right), 6.60$ $\left(\mathrm{d}, 1 \mathrm{H}, J=15.2 \mathrm{~Hz}, \mathrm{C}_{7}-\mathrm{H}\right), 2.62\left(\mathrm{~s}, 3 \mathrm{H}, \mathrm{C}_{3}-\mathrm{CH}_{3}\right), 2.39$ (s, $3 \mathrm{H}, \mathrm{C}_{4^{\prime \prime}}-\mathrm{CH}_{3}$ ), 1.55 (br s, $1 \mathrm{H}, \mathrm{NH}$ ); NMR $\delta_{\mathrm{C}}(100$ $\mathrm{MHz}, \mathrm{CDCl}_{3}$ ): 183.4 (C-6), 165.5 (C-5), 159.1 (C-3), 148.2 (C-8), $142.4\left(\mathrm{C}-4^{\prime \prime}\right), 137.1\left(\mathrm{C}-1^{\prime}\right), 130.9\left(\mathrm{C}-1^{\prime \prime}\right)$, $129.8\left(\mathrm{C}-2^{\prime \prime} / \mathrm{C}-6^{\prime \prime}\right), 129.0\left(\mathrm{C}-3^{\prime \prime} / \mathrm{C}-5^{\prime \prime}\right), 128.9\left(\mathrm{C}-3^{\prime} / \mathrm{C}-\right.$ 5'), $125.9\left(\mathrm{C}-4^{\prime}\right), 119.4\left(\mathrm{C}-2^{\prime} / \mathrm{C}-6^{\prime}\right), 117.6(\mathrm{C}-7), 21.6$ $\left(\mathrm{C}_{4^{\prime \prime}}-\mathrm{CH}_{3}\right), 20.8\left(\mathrm{C}_{3}-\mathrm{CH}_{3}\right)$; HRMS: m/z $\left(\mathrm{M}^{+}\right)$calcd. for $\mathrm{C}_{20} \mathrm{H}_{18} \mathrm{~N}_{2} \mathrm{O}_{2}$ : 318.1363, found: $319.1435(\mathrm{M}+\mathrm{H})$. 
2.3d s-trans (E) 4-(3-(4-Chlorophenyl)acryloyl)-5methyl-2-phenyl-1H-pyrazol-3(2H)-one (3d): M.p.: 160-161 ${ }^{\circ} \mathrm{C}$; yield: 93\%; IR(KBr)v: 3165, 1708, 1591 $\mathrm{cm}^{-1} ; \mathrm{NMR}_{\mathrm{H}}\left(400 \mathrm{MHz}, \mathrm{CDCl}_{3}\right): 7.85(\mathrm{~d}, 1 \mathrm{H}, J=$ $\left.15.6 \mathrm{~Hz}, \mathrm{C}_{8}-\mathrm{H}\right), 7.80\left(\mathrm{~d}, 2 \mathrm{H}, J=8.0 \mathrm{~Hz}, \mathrm{C}_{2^{\prime}}-\mathrm{H}, \mathrm{C}_{6^{\prime}}-\right.$ $\mathrm{H}), 7.43\left(\mathrm{~d}, 2 \mathrm{H}, J=8.4 \mathrm{~Hz}, \mathrm{C}_{2^{\prime \prime}}-\mathrm{H}, \mathrm{C}_{6^{\prime \prime}}-\mathrm{H}\right), 7.37-7.33$ $\left(\mathrm{m}, 4 \mathrm{H}, \mathrm{C}_{3^{\prime}}-\mathrm{H}, \mathrm{C}_{5^{\prime}}-\mathrm{H}, \mathrm{C}_{3^{\prime \prime}}-\mathrm{H}, \mathrm{C}_{5^{\prime \prime}}-\mathrm{H}\right), 7.19$ (t, $1 \mathrm{H}, J=$ $\left.7.2 \mathrm{~Hz}, \mathrm{C}_{4^{\prime}}-\mathrm{H}\right), 6.61\left(\mathrm{~d}, 1 \mathrm{H}, J=15.6 \mathrm{~Hz}, \mathrm{C}_{7}-\mathrm{H}\right)$, $2.62\left(\mathrm{~s}, 3 \mathrm{H}, \mathrm{C}_{3}-\mathrm{CH}_{3}\right), 1.55$ (br s, $\left.1 \mathrm{H}, \mathrm{NH}\right) ; \mathrm{NMR} \delta_{\mathrm{C}}$ $\left(100 \mathrm{MHz}, \mathrm{CDCl}_{3}\right)$ : 183.6 (C-6), 165.3 (C-5), 153.0 (C-3), 146.7 (C-8), 137.7 (C-1'), 137.0 (C-1") 132.0 (C-4'), , $130.1\left(\mathrm{C}-2^{\prime \prime} / \mathrm{C}-6^{\prime \prime}\right), 128.9\left(\mathrm{C}-3^{\prime} / \mathrm{C}-5^{\prime}\right), 126.1$ (C-4'), $119.4\left(\mathrm{C}-2^{\prime \prime} / \mathrm{C}-6^{\prime \prime}\right), 119.0(\mathrm{C}-7), 20.8\left(\mathrm{C}_{3}-\mathrm{CH}_{3}\right)$; HRMS: $\mathrm{m} / \mathrm{z}\left(\mathrm{M}^{+}\right)$calcd. for $\mathrm{C}_{19} \mathrm{H}_{15} \mathrm{ClN}_{2} \mathrm{O}_{2}: 338.0821$, found: $339.0891(\mathrm{M}+\mathrm{H})$.

2.3e s-trans (E) 4-(3-(Furan-2-yl)acryloyl)-5-methyl2-phenyl-1H-pyrazol-3(2H)-one (3e): M.p.: 114$115^{\circ} \mathrm{C}$; yield: $92 \%$; IR(KBr)v: 3126, 1707, 1678, 1598 $\mathrm{cm}^{-1}$; NMR $\delta_{\mathrm{H}}\left(400 \mathrm{MHz}, \mathrm{CDCl}_{3}\right): 7.92(\mathrm{~d}, 2 \mathrm{H}, J=$ $\left.8.0 \mathrm{~Hz}, \mathrm{C}_{2^{\prime}}-\mathrm{H}, \mathrm{C}_{6^{\prime}}-\mathrm{H}\right), 7.61\left(\mathrm{~d}, 1 \mathrm{H}, J=15.6 \mathrm{~Hz}, \mathrm{C}_{8}-\mathrm{H}\right)$, $7.59\left(\mathrm{~s}, 1 \mathrm{H}, \mathrm{C}_{3^{\prime \prime}}-\mathrm{H}\right), 7.33\left(\mathrm{t}, 2 \mathrm{H}, J=8.0,7.6 \mathrm{~Hz}, \mathrm{C}_{3^{\prime}}-\mathrm{H}\right.$, $\left.\mathrm{C}_{5^{\prime}}-\mathrm{H}\right), 7.17\left(\mathrm{t}, 1 \mathrm{H}, J=7.6,7.2 \mathrm{~Hz}, \mathrm{C}_{4^{\prime}}-\mathrm{H}\right), 6.74(\mathrm{~d}$, $\left.1 \mathrm{H}, J=3.2 \mathrm{~Hz}, \mathrm{C}_{5^{\prime \prime}}-\mathrm{H}\right), 6.51\left(\mathrm{~d}, 1 \mathrm{H}, J=15.6 \mathrm{~Hz}, \mathrm{C}_{7^{-}}\right.$ $\mathrm{H}), 6.49$ (br s, $\left.1 \mathrm{H}, \mathrm{C}_{4^{\prime \prime}}-\mathrm{H}\right), 2.62\left(\mathrm{~s}, 3 \mathrm{H}, \mathrm{C}_{3}-\mathrm{CH}_{3}\right), 1.55$ (br s, $1 \mathrm{H}, \mathrm{NH})$; NMR $\delta_{\mathrm{C}}\left(100 \mathrm{MHz}, \mathrm{CDCl}_{3}\right): 183.3(\mathrm{C}-$ 6), 165.5 (C-5), 158.9 (C-3), 150.6 (C-2"), 146.1 (C-8), $137.1\left(\mathrm{C}-1^{\prime}\right), 133.2\left(\mathrm{C}-5^{\prime \prime}\right), 128.8\left(\mathrm{C}-3^{\prime} / \mathrm{C}-5^{\prime}\right), 125.9$ (C-4'), 119.5 (C-2'/C-6'), 118.6 (C-7), 116.1 (C-3"), $113.0\left(\mathrm{C}-4^{\prime \prime}\right), 20.8\left(\mathrm{C}_{3}-\mathrm{CH}_{3}\right)$; HRMS: m/z $\left(\mathrm{M}^{+}\right)$calcd. for $\mathrm{C}_{17} \mathrm{H}_{14} \mathrm{~N}_{2} \mathrm{O}_{3}$ : 294.1003, found: $295.107(\mathrm{M}+\mathrm{H})$.

$2.3 f \quad s$-trans (E) 4-(3-(4-Bromophenyl)acryloyl)-5methyl-2-phenyl-1H-pyrazol-3(2H)-one (3f): M.p.: 135-136 ${ }^{\circ} \mathrm{C}$ yield: $98 \%$; IR(KBr)v: 3068, 1708, 1597 , $1489 \mathrm{~cm}^{-1}$; NMR $\delta_{\mathrm{H}}\left(400 \mathrm{MHz}, \mathrm{CDCl}_{3}\right): 7.83(\mathrm{~d}, 1 \mathrm{H}$, $\left.J=15.3 \mathrm{~Hz}, \mathrm{C}_{8}-\mathrm{H}\right), 7.80\left(\mathrm{~d}, 2 \mathrm{H}, J=8.6 \mathrm{~Hz}, \mathrm{C}_{2^{\prime}}\right.$, $\left.\mathrm{H}, \mathrm{C}_{6^{\prime}}-\mathrm{H}\right), 7.51\left(\mathrm{~d}, 2 \mathrm{H}, J=8.4 \mathrm{~Hz}, \mathrm{C}_{3^{\prime \prime}}-\mathrm{H}, \mathrm{C}_{5^{\prime \prime}}-\mathrm{H}\right)$, 7.37-7.33 (m, 4H, C $\left.\mathrm{3}_{3^{\prime}}-\mathrm{H}, \mathrm{C}_{5^{\prime}}-\mathrm{H}, \mathrm{C}_{2^{\prime \prime}}-\mathrm{H}, \mathrm{C}_{6^{\prime \prime}}-\mathrm{H}\right), 7.19$ $\left(\mathrm{t}, 1 \mathrm{H}, J=7.4,6.8 \mathrm{~Hz}, \mathrm{C}_{4^{\prime}}-\mathrm{H}\right), 6.62(\mathrm{~d}, 1 \mathrm{H}, J=15.3$ $\left.\mathrm{Hz}, \mathrm{C}_{7}-\mathrm{H}\right), 2.59$ (s, 3H, $\mathrm{C}_{3}-\mathrm{CH}_{3}$ ), 1.55 (br s, $1 \mathrm{H}, \mathrm{NH}$ ); NMR $\delta_{\mathrm{C}}\left(100 \mathrm{MHz}, \mathrm{CDCl}_{3}\right): 183.3$ (C-6), $165.2(\mathrm{C}-5)$, 158.6 (C-3), 146.7 (C-8), 137.0 (C-1'), 132.5 (C-3"/C$\left.5^{\prime \prime}\right), 132.4\left(\mathrm{C}-1^{\prime \prime}\right), 130.2\left(\mathrm{C}-2^{\prime \prime} / \mathrm{C}-6^{\prime \prime}\right), 128.9\left(\mathrm{C}-3^{\prime} / \mathrm{C}-5^{\prime}\right)$, $126.2\left(\mathrm{C}-4^{\prime \prime}\right), 126.1$ (C-4') $119.4\left(\mathrm{C}-2^{\prime} / \mathrm{C}-6^{\prime}\right), 119.1$ (C-7), $20.8\left(\mathrm{C}_{3}-\mathrm{CH}_{3}\right)$; HRMS: $\mathrm{m} / \mathrm{z}\left(\mathrm{M}^{+}\right)$calcd. for $\mathrm{C}_{19} \mathrm{H}_{15} \mathrm{BrN}_{2} \mathrm{O}_{2}$ : 382.0313 , found: $383.0384(\mathrm{M}+\mathrm{H})$.

\subsection{Evaluation of cytotoxicity assay}

All the pyrazolyl chalcones i.e.s-cis (E)-1-(5-hydroxy3-methyl-1-phenyl-1 $H$-pyrazol-4-yl)-3-arylprop-2-en- 1-one (2) and s-trans (E)-4-(3-(phenyl/aryl/hetroaryl) acryloyl)-5-methyl-2-phenyl-1 $H$-pyrazol-3(2H)-ones (3) were dissolved in DMSO to form stock solutions $(10 \mathrm{mM} / \mathrm{mL})$, which were filter sterilized before testing on cell lines. Human Pancreatic cancer cell line (PANC1), Colon (Colo-205, HCT-116), lung carcinoma cell line (A549, NCI-H322) were procured from European Collection of cell cultures (ECACC). The human cancer cell lines were grown in tissue culture flasks in complete growth medium (RPMI-1640/ MEM/ DMEM medium supplemented with $10 \%$ fetal calf serum, $100 \mu \mathrm{g} / \mathrm{mL}$ streptomycin and 100 units/mL penicillin) in carbon dioxide incubator (New Brunswick, Galaxy $170 \mathrm{R}$, Eppendorf) at $37^{\circ} \mathrm{C}, 5 \% \mathrm{CO}_{2}$ and $98 \%$ $\mathrm{RH}$.

The sulforhodamine B (SRB) assay was performed, in which cell suspension of optimum cell density (7500-15000 cells/100 $\mu \mathrm{L}$ ) was seeded and exposed to $1 \mu \mathrm{M}, 10 \mu \mathrm{M}, 30 \mu \mathrm{M}, 50 \mu \mathrm{M}$, concentrations of test materials in complete growth medium $(100 \mu \mathrm{L})$ were added after $24 \mathrm{~h}$ of incubation along with known cytotoxic agents paclitaxel, 5-Florouracil (5-FU) and Erlotinib as positive controls. After further $48 \mathrm{~h}$, incubation cells were fixed with ice-cold TCA for $1 \mathrm{~h}$ at $4^{\circ} \mathrm{C}$. After $1 \mathrm{~h}$, the plates were washed five times with distilled water and allowed to air dry followed by the addition of $100 \mu \mathrm{L}$ of $0.4 \%$ SRB dye for $0.5 \mathrm{~h}$ at room temperature. Plates were then washed with $1 \%$ $\mathrm{v} / \mathrm{v}$ acetic acid to remove the unbound SRB. The bound dye was solubilized by adding $100 \mu \mathrm{L}$ of $10 \mathrm{mM}$ tris buffer $(\mathrm{pH}=10.4)$ to each well. The plates were put on the shaker for $5 \mathrm{~min}$ to solublize the dye completely, and finally the reading was taken at $540 \mathrm{~nm}$ on microplate reader (BioTek Synergy HT). $\mathrm{IC}_{50}$ was determined by plotting OD against concentration. ${ }^{14}$

\subsection{Evaluation of anti-microbial activity (determination of MIC)}

The anti-microbial activity of all the pyrazolyl chalcones ( $\mathbf{2}$ and $\mathbf{3}$ ) was performed against Gram-positive bacteria: Staphylcococcus aureus [MTCC 2901], Bacillus sublitis [MTCC 2063], Gram-negative bacterium: Escherichia coli [MTCC 1652] and fungal strains: Candida albicans [MTCC 227] and Aspergillus niger [MTCC 8189] using tube dilution method. Dilutions of test and standard compounds were prepared in double strength nutrient broth - I.P. (bacteria) or Sabouraud dextrose broth I.P. (fungi). The samples were incubated at $37^{\circ} \mathrm{C} \pm 1^{\circ} \mathrm{C}$ for $24 \mathrm{~h}$ (bacteria), $25^{\circ} \mathrm{C}$ for 7 days (A. niger) and $37^{\circ} \mathrm{C} \pm 1^{\circ} \mathrm{C}$ for $48 \mathrm{~h}$ (C. albicans) and the results were recorded in terms of MIC. 


\section{Results and Discussion}

\subsection{Chemistry}

The 4-acylpyrazolones are interesting class of $\beta$ diketones containing pyrazole fused to a chelating arm that is useful in coordination chemistry. ${ }^{15}$ In the present study, 1-phenyl-3-methyl-4-acetylpyrazol-5-one $(\mathbf{1})^{16}$ is used as starting material for the synthesis of cis (E)-1-(5-hydroxy-3-methyl-1-phenyl-1 $H$-pyrazol4-yl)-3-phenyl/aryl/heteroarylprop-2-en-1-ones (2, OH form) in good yield (70-88\%) by condensation with aromatic aldehydes (scheme 1). It may be mentioned that some of pyrazolyl chalcones (2) have been prepared using different reaction conditions in low yield (30-40\%) without reporting any spectroscopic data and explanation about structure. ${ }^{13}$ However, all the pyrazolyl chalcones have been synthesized with improved procedure.

The pyrazolyl chalcones (2) were subjected to rigorous study as they can adopt different conformations by rotating around the sigma bond of enone moiety leading to different geometries: $E$ s-cis and s-trans, $Z$ s-cis and $s$-trans conformations along with enol ( $\mathrm{OH}$ and keto) and $(\mathrm{NH})$ tautomerism (figures 1 and 2). The structure of compound $\mathbf{2}$ was determined with the help of IR, NMR $\left({ }^{1} \mathrm{H}\right.$ and ${ }^{13} \mathrm{C}$ ), 2D-NMR (COSY, HSQC, HMBC, TOCSY and ROESY) and HRMS data. The IR spectrum of $\mathbf{2 f}$ showed the bands at $3059,2927,1631 \mathrm{~cm}^{-1}$ due to $\mathrm{C}-\mathrm{H}$ str. and $\mathrm{C}=\mathrm{O}$ str. The $\mathrm{O}-\mathrm{H} / \mathrm{N}-\mathrm{H}$ str. vibrational band was not observed. In ${ }^{1} \mathrm{H}$ NMR spectrum, the two doublets of enone moiety appeared at $\delta 7.13$ $(J=15.6 \mathrm{~Hz})$ and $7.85(J=15.6 \mathrm{~Hz})$ assigned to $\mathrm{C}_{7}-\mathrm{H}$ $\left(\mathrm{H}_{\alpha}\right)$ and $\mathrm{C}_{8}-\mathrm{H}\left(\mathrm{H}_{\beta}\right)$ respectively, indicate thereby the $E$ configuration around the double bond of enone moiety. The pyrazolyl chalcone (2f) having $Z$ configuration was not observed at all. This fact is also supported by the literature study indicating that the chalcone having $E$ configuration is the principle product of reaction between aldehyde and ketone. ${ }^{17}$ The ${ }^{13} \mathrm{C}$ NMR of $\mathbf{2 f}$ displayed signals due to enone moiety at $\delta 177.9$, 119.8 and 142.6 assigned to $\mathrm{C}=\mathrm{O}, \mathrm{C}-7$ and $\mathrm{C}-8$, respectively. The high resolution mass spectrum of $\mathbf{2 f}$ showed the molecular ion peak at $\mathrm{m} / \mathrm{z} 383.0389(\mathrm{M}+\mathrm{H})$ (calcd. for $\mathrm{C}_{19} \mathrm{H}_{15} \mathrm{BrN}_{2} \mathrm{O}_{2}$ : 382.0315).

Further, pyrazolyl chalcones (2) may exist more as $\mathrm{OH}$ and $\mathrm{NH}$ tautomer (figures 1 and 2) due to fast protropic exchange and may involve intramolecular hydrogen bonding. The $\mathrm{OH} / \mathrm{NH}$ signal in NMR could not be observed. Further, 2 is also having s-cis $(E)$ geometry rather than the s-trans $(E)$ geometry based on detailed NMR analysis. It was earlier noted that two double bonds of enone moiety in chalcones were positioned cis with respect to each other in several x-ray crystal structure of chalcone and the s-cis isomer of chalcone was more stable that the $s$-trans conformer by at least $3.9 \mathrm{kcal} / \mathrm{mol} .{ }^{18}$ Each mutually coupled protons and their connectivities with carbons along with identification of different carbons and long range coupling were analysed thoroughly with the help of COSY, HSQC, DEPT135 and HMBC experiments for structure elucidation of pyrazolyl chalcone (2).

The cis (E)-1-(5-hydroxy-3-methyl-1-phenyl-1Hpyrazol-4-yl)-3-phenyl/aryl/heteroarylprop-2-en-1-ones (2) was stirred in dichloromethane at room temperature in presence of iodobenzene diacetate. To our surprise, 2 was isomerized to s-trans (E)-4-(3-(phenyl/aryl/ hetroaryl)acryloyl)-5-methyl-2-phenyl- $1 H$-pyrazol-3(2H)ones (3, NH form) instead of the formation of pyranopyrazole (4) (scheme 2). The structure of 3 was determined using IR, NMR $\left({ }^{1} \mathrm{H}\right.$ and $\left.{ }^{13} \mathrm{C}\right)$, DEPT-135, 2DNMR (COSY, HSQC, HMBC, TOCSY and ROESY), NOE difference spectra and HRMS data. The IR spectrum of $\mathbf{3 f}$ showed the bands at 3068 and $1708 \mathrm{~cm}^{-1}$ due to $\mathrm{C}-\mathrm{H}$ str. and $\mathrm{C}=\mathrm{O}$ str., respectively. However, $\mathrm{O}-\mathrm{H} / \mathrm{N}-\mathrm{H}$ str. vibrational band was not observed. In ${ }^{1} \mathrm{H}$ NMR spectrum, the two doublets of enone moiety appeared at $\delta 6.62(J=15.3 \mathrm{~Hz})$ and $7.83(J=15.3 \mathrm{~Hz})$

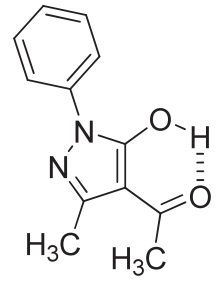

1

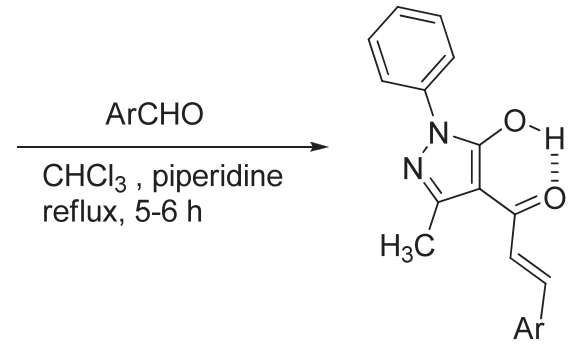

2

$\mathrm{Ar}=\mathrm{a}, \mathrm{C}_{6} \mathrm{H}_{5} ; \mathrm{b}, \mathrm{C}_{6} \mathrm{H}_{4} \mathrm{OCH}_{3}(4) ; \mathrm{c}, \mathrm{C}_{6} \mathrm{H}_{4} \mathrm{CH}_{3}(4) ; \mathrm{d} \mathrm{C}_{6} \mathrm{H}_{4} \mathrm{Cl}(4) ; \mathrm{e}$, furyl; f, $\mathrm{C}_{6} \mathrm{H}_{4} \mathrm{Br}(4)$

Scheme 1. Synthesis of cis (E)-1-(5-hydroxy-3-methyl-1-phenyl-1H-pyrazol4-yl)-3-phenyl/aryl/heteroarylprop-2-en-1-ones (2). 


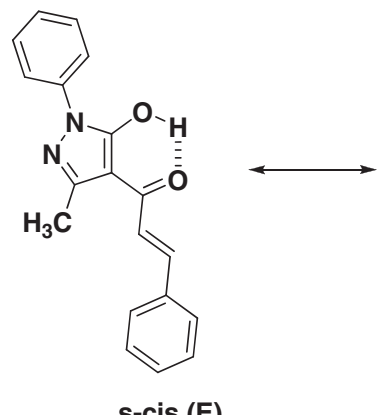

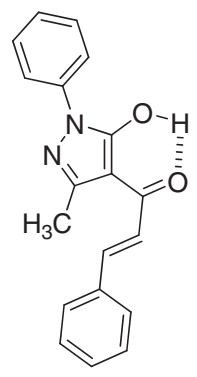

s-trans (E)

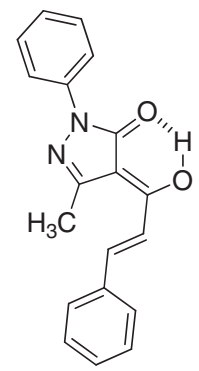

s-cis (E)

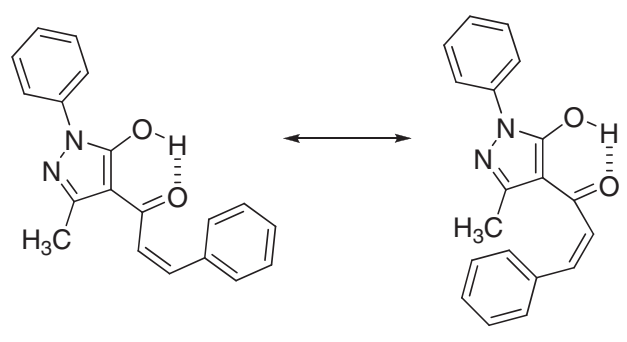

s-cis (Z)

s-trans (Z)

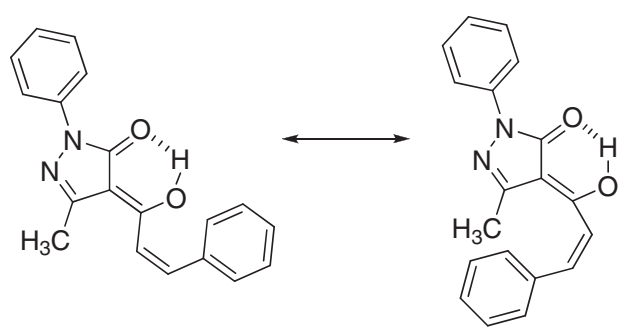

s-trans (Z)

S-cis $(Z)$

Figure 1. $\mathrm{OH}$ tautomer of $\mathbf{2}$.

assigned to $\mathrm{C}_{7}-\mathrm{H}\left(\mathrm{H}_{\alpha}\right)$ and $\mathrm{C}_{8}-\mathrm{H}\left(\mathrm{H}_{\beta}\right)$, respectively indicating thereby again the $E$ configuration around sigma bond of enone moiety. But the $\mathrm{C}_{7}-\mathrm{H}\left(\mathrm{H}_{\alpha}\right)$ was found to be shielded in comparison to $\mathrm{C}_{7}-\mathrm{H}\left(\mathrm{H}_{\alpha}\right)$ of 2 that resonated at $\delta 7.13$. The $\mathrm{NH}$ signal was observed at $\delta 1.55$. The ${ }^{13} \mathrm{C}$ NMR of $\mathbf{3 f}$ displayed signals due to enone moiety at $\delta 183.3,119.1$ and 146.7 assigned to $\mathrm{C}=\mathrm{O}$, $\mathrm{C}-7$ and $\mathrm{C}-8$, respectively. The high resolution mass spectrum of $3 \mathbf{f}$ showed the molecular ion peak at $\mathrm{m} / \mathrm{z}$ 383.0384 ( $\mathrm{M}+\mathrm{H})$ (calcd. for $\mathrm{C}_{19} \mathrm{H}_{15} \mathrm{BrN}_{2} \mathrm{O}_{2}:$ 382.0315).

The isomerization of $s$-cis geometry to s-trans geometry around sigma bond was established by analysis of the data presented in table 1 . There was almost no change in the chemical shift value of proton $\left(\mathrm{C}_{8}-\mathrm{H}\right)$ in both 2 and 3 , but the proton $\left(\mathrm{C}_{7}-\mathrm{H}\right)$ is shielded $(\sim 0.5 \mathrm{ppm})$ in the product $(3)$ in comparison to the chemical shift value of the proton $\left(\mathrm{C}_{7}-\mathrm{H}\right)$ in the reactant (2). Further, it was observed that there was very small deshielding $(\sim 0.04 \mathrm{ppm})$ of methyl proton $\left(\mathrm{C}_{3}-\mathrm{CH}_{3}\right)$ of pyrazole moiety. The melting point of the products (3) is also lower in comparison to the reactants (2) suggesting a decrease in the extent of hydrogen bonding. The role of pyrazole moiety in isomerization cannot be ruled out because when $(E)$-1-(2-hydroxyphenyl)-3-arylprop2-en-1-ones were treated with iodobenzene diacetate under similar condition there was no isomerization, rather the starting material recovered back.

There was difference in the chemical shift values of carbons C-3, C-6 and C-7 in compounds 2 and 3 as indicated in the table 2 . The increase in chemical shift value by $\sim 12$ ppm C-3 of pyrazole moiety and by $\sim 5 \mathrm{ppm}$ in $\mathrm{C}-6(\mathrm{C}=\mathrm{O})$ and $\mathrm{C}-7$ of enone moiety was observed on

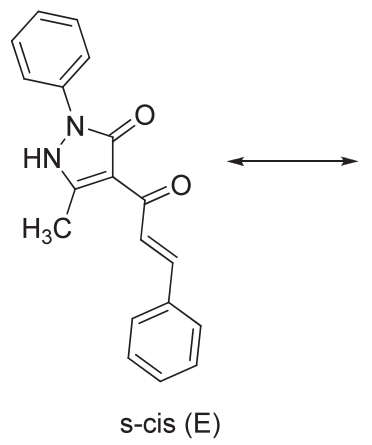

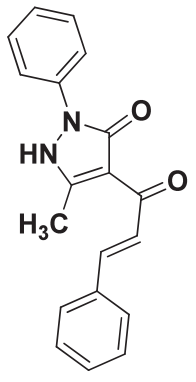

s-trans (E)

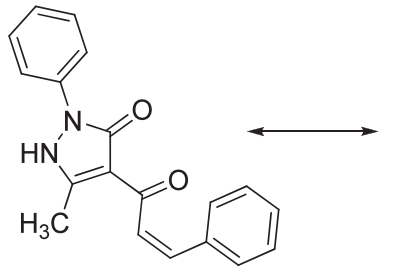

s-cis $(Z)$

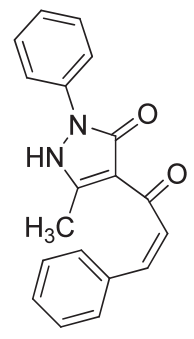

s-trans (Z)

Figure 2. NH tautomer of 2. 


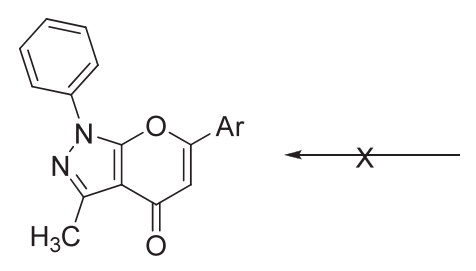

4



2

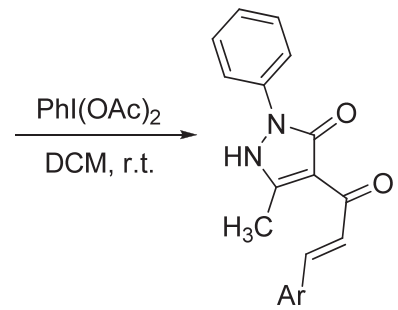

3

$\mathrm{Ar}=\mathrm{a}, \mathrm{C}_{6} \mathrm{H}_{5} ; \mathrm{b}, \mathrm{C}_{6} \mathrm{H}_{4} \mathrm{OCH}_{3}(4) ; c, \mathrm{C}_{6} \mathrm{H}_{4} \mathrm{CH}_{3}(4) ; \mathrm{d} \mathrm{C}_{6} \mathrm{H}_{4} \mathrm{Cl}(4) ; \mathrm{e}$, furyl; f, $\mathrm{C}_{6} \mathrm{H}_{4} \mathrm{Br}(4)$

Scheme 2. Isomerization of $s$-trans $(E)$-4-(3-(phenyl/aryl/heteroaryl)acryloyl)5-methyl-2-phenyl-1 $H$-pyrazol-3( $2 H)$-ones (3).

isomerization of $\mathbf{2}$ to $\mathbf{3}$. However, there was no difference in chemical shift values of carbons $\mathrm{C}-5(\mathrm{C}=\mathrm{O})$ and very minor difference in chemical shift of C-8. Moreover, the changes in the chemical shift value of carbon (C-3) of pyrazole moiety form $\delta 146.9$ to 158.6 might be expected only when $\mathrm{OH}$ tautomer of pyrazole is transformed to $\mathrm{NH}$ tautomer. ${ }^{19}$ These data support the isomerization of $\mathbf{2}$ to $\mathbf{3}$. However, the C-4 chemical shift value was not observed. The s-cis $(E)$ geometry of $\mathbf{2}$ and $s$-trans $(E)$ geometry of $\mathbf{3}$ was also supported by TOCSY, ROESY and 1D NOE difference spectra as explained for $\mathbf{2 f}$ and $\mathbf{3 f}$ (figure 3). TOCSY and ROESY experiments established the correlation of hydrogens in space and the percentage enhancement of signals in 1D NOE difference spectrum explained the proximity of hydrogens in space thereby substantiating the above assignment in support of the structure of $\mathbf{2} \mathbf{f}$ and $\mathbf{3 f}$.

In 1D NOE difference spectra of $\mathbf{2 f}$, saturating $\mathrm{C}_{7}-\mathrm{H}$ showed unexpected enhancement to $\mathrm{C}_{3}-\mathrm{CH}_{3}(37.4 \%)$ of pyrazole moiety whereas saturating $\mathrm{C}_{3}-\mathrm{CH}_{3}$ gave $2.39 \%$ enhancement of $\mathrm{C}_{7}-\mathrm{H}$. Saturating $\mathrm{C}_{8}-\mathrm{H}$ showed only enhancement to $\mathrm{C}_{2^{\prime \prime}} / 6^{\prime \prime}-\mathrm{H}(0.43 \%)$. In case of 3f, saturating $\mathrm{C}_{3}-\mathrm{CH}_{3}$ of pyrazole moiety indicated enhancement to $\mathrm{C}_{-2^{\prime} / 6^{\prime}}-\mathrm{H}(0.36 \%), \mathrm{C}_{7}-\mathrm{H}(1.27 \%)$ and $\mathrm{C}_{8}-\mathrm{H}(0.29 \%)$ whereas saturating $\mathrm{C}_{8}-\mathrm{H}$ produced enhancement of $\mathrm{C}_{3}-\mathrm{CH}_{3}$ (1.36\%), $\mathrm{C}_{7}-\mathrm{H}(0.92 \%)$ and $\mathrm{C}_{2^{\prime \prime} / 6^{\prime \prime}}-\mathrm{H}(2.39 \%)$. Further, saturating $\mathrm{C}_{7}-\mathrm{H}$ shows only enhancement in $\mathrm{C}_{2^{\prime \prime} / 6^{\prime \prime}}-\mathrm{H}(0.44 \%)$. All these results are consistent with the $s$-cis $(E)$ geometry for $\mathbf{2 f}$ and $s$-trans (E) geometry for $\mathbf{3 f}$.
The probable mechanism consists of addition of iodobenzene diacetate on enone double bond of $\mathbf{2}$ resulting in the formation of $\mathbf{5}$ that may undergo rotation around single bond thus producing $\mathbf{6}$ thereby relieving the steric strain. The intermediate $\mathbf{6}$ is then attacked by the moisture to form the cyclic intermediate (7) that eliminates iodosobenzene to produce $\mathbf{3}$. However, the pyranopyrazole (4) was not formed even in traces (scheme 3). In order to establish the role of moisture, the above reaction was also carried out in dichloromethane in presence of a drop of water; the result was found to be same.

\subsection{Biological studies}

3.2a Cytotoxic activity: The cytotoxic activity of $s$-cis (E)-1-(5-hydroxy-3-methyl-1-phenyl-1 $H$-pyrazol4-yl)-3-arylprop-2-en-1-ones (2) and $s$-trans (E)-1-(5hydroxy-3-methyl-1-phenyl-1 $H$-pyrazol-4-yl)-3-arylprop2-en-1-ones (3) based on SRB (Sulphorhodamine B assay) was performed against panel of five cancer cell lines (PANC-1, COLO-205, HCT-116, A549 and NCIH322). The cytotoxicity of pyrazolyl chalcone (2c) was found to be more active at very low concentrations with $\mathrm{IC}_{50}$ of $13.3 \mu \mathrm{M}$ against colon cancer cell line (HCT-116) and it is moderately active against A549 cancer cell line. However, pyrazolyl chalcones ( $\mathbf{2 d}$ and 2e) were also active against $\mathrm{A} 549$ with $\mathrm{IC}_{50}$ values of 25.4 and $23.0 \mu \mathrm{M}$, respectively whereas $\mathbf{2 d}$ was also active against NCI-H322 with $\mathrm{IC}_{50} 25.7 \mu \mathrm{M}$. These

Table 1. Melting point and selected proton chemical shift values of $\mathbf{2}$ and $\mathbf{3}$.

\begin{tabular}{lccccccc}
\hline Compd. & M.p. $\left({ }^{\circ} \mathrm{C}\right)$ & $\delta\left(\mathrm{H}_{\alpha}\right)$ & $\delta\left(\mathrm{H}_{\beta}\right)$ & Compd. & M.p. $\left({ }^{\circ} \mathrm{C}\right)$ & $\delta\left(\mathrm{H}_{\alpha}\right)$ & $\delta\left(\mathrm{H}_{\beta}\right)$ \\
\hline $\mathbf{2 a}$ & $158-159$ & 7.16 & 7.94 & 3a & $142-143$ & 6.65 & 7.92 \\
$\mathbf{2 b}$ & $152-153$ & 7.01 & 7.91 & 3b & $140-141$ & 6.52 & 7.87 \\
$\mathbf{2 c}$ & $158-159$ & 7.11 & 7.88 & $\mathbf{3 c}$ & $144-145$ & 6.60 & 7.89 \\
$\mathbf{2 d}$ & $171-172$ & 7.15 & 7.90 & $\mathbf{3 d}$ & $160-161$ & 6.61 & 7.85 \\
$\mathbf{2 e}$ & $168-169$ & 7.01 & 7.66 & $\mathbf{3 e}$ & $114-115$ & 6.51 & 7.61 \\
$\mathbf{2 f}$ & $169-170$ & 7.13 & 7.85 & $\mathbf{3 f}$ & $135-136$ & 6.62 & 7.83 \\
\hline
\end{tabular}


Table 2. Selected carbon shift values of compounds $\mathbf{2}$ and $\mathbf{3}$.

\begin{tabular}{lcccccc}
\hline Compound & C-3 & C-4 & C-5 & C-6 & C-7 & C-8 \\
\hline $\mathbf{2 a}$ & 147.0 & 104.7 & 165.2 & 178.0 & 119.2 & 144.1 \\
$\mathbf{2 b}$ & 147.0 & 104.3 & 165.6 & 177.6 & 116.4 & 144.0 \\
$\mathbf{2 c}$ & 147.0 & 104.4 & 165.5 & 177.8 & 118.0 & 144.2 \\
$\mathbf{2 d}$ & 146.9 & 104.7 & 165.0 & 177.9 & 119.8 & 142.5 \\
$\mathbf{2 e}$ & 151.3 & 104.8 & 165.5 & 177.0 & 117.3 & 145.8 \\
$\mathbf{2 f}$ & 146.9 & 104.8 & 165.0 & 177.9 & 119.8 & 142.6 \\
$\mathbf{3 a}$ & 158.9 & n.o. & 165.4 & 183.4 & 118.6 & 148.1 \\
$\mathbf{3 b}$ & 159.2 & n.o. & 165.7 & 183.3 & 116.1 & 147.8 \\
$\mathbf{3 c}$ & 159.1 & n.o. & 165.5 & 183.4 & 117.6 & 148.2 \\
$\mathbf{3 d}$ & 153.0 & n.o. & 165.3 & 183.6 & 119.0 & 146.7 \\
$\mathbf{3 e}$ & 158.9 & n.o. & 165.5 & 183.3 & 118.6 & 146.1 \\
$\mathbf{3 f}$ & 158.6 & n.o. & 165.2 & 183.3 & 119.1 & 146.7 \\
\hline
\end{tabular}

n.o. not observed

result depicted that prazolyl cahlcones (2) having s-cis configuration are more active than the isomeric pyrazolyl chalcones (3) having s-trans configuration except 3a as reflected by relative $\mathrm{IC}_{50}$ values (table 3 ).

3.2b Anti-microbial activity: The s-cis (E)-1-(5-hydroxy3-methyl-1-phenyl-1 $H$-pyrazol-4-yl)-3-arylprop-2-en-1ones (2) and s-trans (E)-4-(3-(phenyl/aryl/hetroaryl)- acryloyl)-5-methyl-2-phenyl-1 $H$-pyrazol-3(2H)-ones (3) were also tested in vitro for their anti-bacterial activity against Gram-positive Bacillus subtilis [MTCC 2063], Staphylococcus aureus [MTCC 2901] and Gram-negative Escherichia coli [MTCC 1652] and in vitro anti-fungal activity against Candida albicans [MTCC 227] and Aspergillus niger [MTCC 8184]. Double strength nutrient broth-I.P. and Sabouraud dextrose broth-I.P ${ }^{20}$ were employed for bacterial



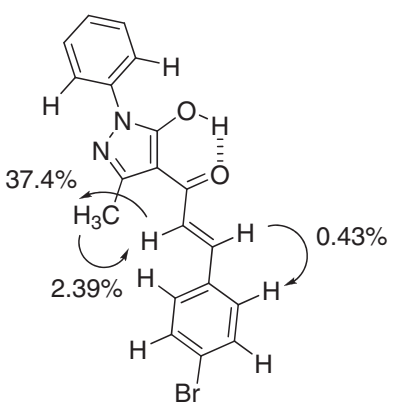

$2 f$

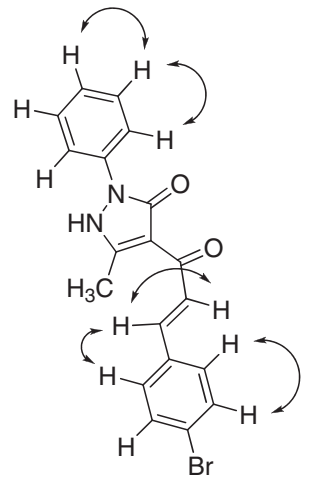

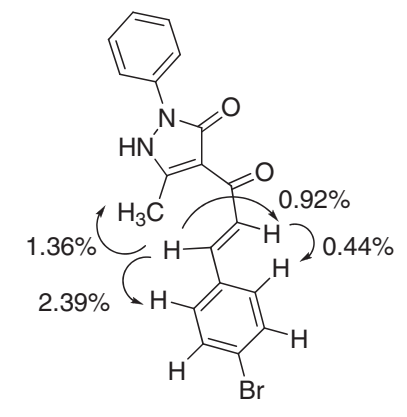

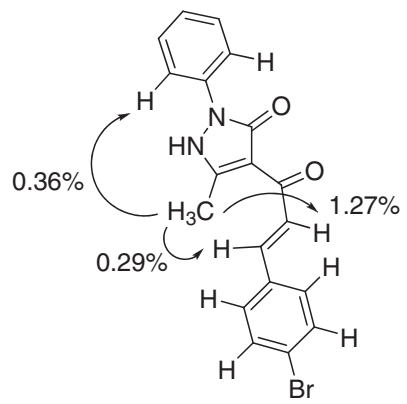

$3 f$

Figure 3. Correlation of hydrogens using TOCSY, ROESY and 1D NOE difference spectra. 


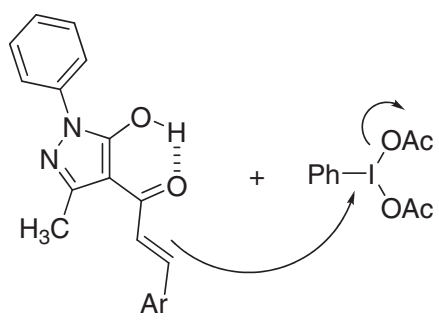

2<smiles>Cc1nn(-c2ccccc2)c2oc(Br)cc(=O)c12</smiles>

4

<smiles>Cc1nn(-c2ccccc2)c2c1C(=O)OC2</smiles>

$-\mathrm{PhIO}, \mathrm{AcOH}$ 3

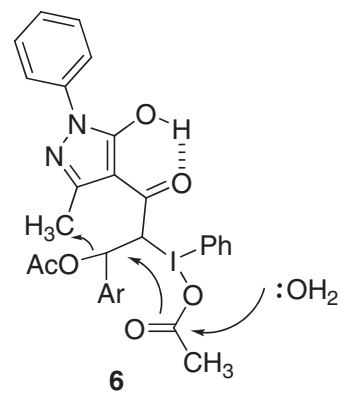



Scheme 3. Mechanism of isomerization of 2 to 3.

and fungal growth respectively. Minimum inhibitory concentrations [MIC] were determined by means of standard serial dilution ${ }^{21}$ and are presented in table 4.

Results of anti-microbial activity (table 4) demonstrated that compound $\mathbf{2 d}$ and $\mathbf{3 d}$ were the most potent ones among the synthesized compounds against $S$. aureus and B. subtilis (MIC range $=0.89-1.85$ $\left.\times 10^{-2} \mu \mathrm{M} / \mathrm{mL}\right)$. Compounds 2d $\left(\mathrm{MIC}_{\mathrm{ec}}=0.92 \times\right.$ $10^{-2} \mu \mathrm{M} / \mathrm{ml}$ ) was found to be most active against $E$. coli. Compound $2 \mathbf{b}\left(\mathrm{MIC}_{\mathrm{ca}}=1.87 \times 10^{-2} \mu \mathrm{M} / \mathrm{ml}\right.$;
$\left.\mathrm{MIC}_{\mathrm{an}}=0.93 \times 10^{-2} \mu \mathrm{M} / \mathrm{ml}\right)$ and $\mathbf{3 b}\left(\mathrm{MIC}_{\mathrm{ca}}=0.93 \times\right.$ $\left.10^{-2} \mu \mathrm{M} / \mathrm{ml} ; \mathrm{MIC}_{\mathrm{an}}=0.93 \times 10^{-2} \mu \mathrm{M} / \mathrm{ml}\right)$ emerged as most active ones against $C$. albicans and A. niger.

Compound 2d having anti-microbial activity close to the standard drug ciprofloxacin may be taken as lead compound for the development of anti-bacterial agents. Similarly compounds $\mathbf{2} \mathbf{b}$ and $\mathbf{3 b}$ can be selected as lead compounds for the development of anti-fungal activity as their activity is close to the standard drug fluconazole.

Table 3. In vitro cytotoxicity $\left(\mathrm{IC}_{50} \mu \mathrm{M}\right)$ of $\mathbf{2}$ and $\mathbf{3}$ against a panel of five human cancer lines.

\begin{tabular}{lccccc}
\hline $\begin{array}{l}\text { Tissue } \rightarrow \\
\text { Cell line } \rightarrow \\
\text { Entry } \downarrow\end{array}$ & $\begin{array}{c}\text { Pancreatic } \\
\text { PANC-1 }\end{array}$ & $\begin{array}{c}\text { Colon } \\
\text { COLO-205 }\end{array}$ & $\begin{array}{c}\text { Colon } \\
\text { HCT-116 } \\
\text { IC } 50\end{array}$ & $\begin{array}{c}\text { Lung } \\
\text { A549 }\end{array}$ & $\begin{array}{c}\text { Lung } \\
\text { NCI-H322 }\end{array}$ \\
\hline $\mathbf{2 a}$ & $>50$ & $>50$ & $>50$ & 49.4 & 35.9 \\
$\mathbf{2 b}$ & $>50$ & $>50$ & 44.2 & 40.8 & $>50$ \\
$\mathbf{2 c}$ & 39.3 & 48.6 & 13.3 & 21.9 & 45.3 \\
$\mathbf{2 d}$ & 37.8 & $>50$ & 30.9 & 25.4 & 25.7 \\
$\mathbf{2 e}$ & 32.2 & 40.6 & 33.3 & 23.0 & 28.2 \\
$\mathbf{2 f}$ & 44.6 & $>50$ & $>50$ & 49.9 & $>50$ \\
$\mathbf{3 a}$ & 34.3 & 26.7 & 32.6 & 30.0 & 48.8 \\
$\mathbf{3 b}$ & $>50$ & $>50$ & $>50$ & 45.3 & $>50$ \\
$\mathbf{3 c}$ & $>50$ & $>50$ & 45.1 & 35.1 & 32.8 \\
$\mathbf{3 d}$ & $>50$ & $>50$ & $>50$ & $>50$ & $>50$ \\
$\mathbf{3 e}$ & $>50$ & $>50$ & $>50$ & $>50$ & $>50$ \\
$\mathbf{3 f}$ & $>50$ & $>50$ & na & na & na \\
Paclitaxel & 0.06 & - & - & 0.2 & - \\
$\mathbf{5 F U}$ & - & 3.2 & 18.8 & - & - \\
Erlotinib & - & - & - & - & 0.35 \\
\hline
\end{tabular}

na $=$ not active 
Table 4. In vitro anti-microbial activity of $\mathbf{2}$ and $\mathbf{3}$.

\begin{tabular}{lccccc}
\hline & \multicolumn{5}{c}{ MIC $\left(10^{-2} \mu \mathrm{M} / \mathrm{mL}\right)$} \\
\cline { 2 - 6 } Compounds & S. aureus & B. subtilis & E. coli & C. albicans & A. niger \\
\hline $\mathbf{2 a}$ & 4.11 & 8.21 & 4.11 & 16.43 & 4.11 \\
$\mathbf{2 b}$ & 7.48 & 3.74 & 7.48 & 1.87 & 0.93 \\
$\mathbf{2 c}$ & 3.93 & 3.93 & 3.93 & 7.85 & 3.93 \\
$\mathbf{2 d}$ & 1.84 & 1.84 & 0.92 & 7.38 & 7.38 \\
$\mathbf{2 e}$ & 4.25 & 8.49 & 4.25 & 16.99 & 4.25 \\
$\mathbf{2 f}$ & 4.46 & 4.46 & 8.92 & 4.46 & 17.84 \\
$\mathbf{3 a}$ & 4.11 & 4.11 & 16.43 & 4.11 & 4.11 \\
$\mathbf{3 b}$ & 7.48 & 3.74 & 7.48 & 0.93 & 0.93 \\
$\mathbf{3 c}$ & 15.70 & 7.85 & 3.93 & 7.85 & 3.93 \\
$\mathbf{3 d}$ & 1.85 & 1.85 & 3.70 & 3.70 & 3.70 \\
$\mathbf{3 e}$ & 4.25 & 4.25 & 4.25 & 8.50 & 17.00 \\
$\mathbf{3 f}$ & 6.77 & 3.39 & 6.77 & 13.54 & 6.77 \\
Std. & $0.94^{\mathrm{a}}$ & $0.94^{\mathrm{a}}$ & $0.94^{\mathrm{a}}$ & $1.02^{\mathrm{b}}$ & $1.02^{\mathrm{b}}$ \\
\hline
\end{tabular}

${ }^{\mathrm{a} C \text { Ciprofloxacin }}{ }^{\mathrm{b}}$ Fluconazole

3.2c Structure activity relationship (SAR) studies: The following structure activity relationship may be drawn from cytotoxic and anti-microbial activity results of $s$-cis (E)-1-(5-hydroxy-3-methyl-1-phenyl-1 $H$ pyrazol-4-yl)-3-arylprop-2-en-1-ones (2) and s-trans (E)-4-(3-(phenyl/aryl/hetroaryl)acryloyl)-5-methyl-2phenyl-1 $H$-pyrazol-3(2H)-ones (3) derivatives:

1. The cytotoxicity screening results indicated that the pyrazolyl ketones (2) are more active than the isomeric pyrazolyl ketones (3) and the presence of methyl group improved the cytotoxic activity of $\mathbf{2 b}$ to the extent that it was better than the control.

2. The presence of electron withdrawing chloro group at $p$-position of the phenyl nucleus attached to position-4 of pyrazole through enone moiety improved the anti-bacterial activity of the pyrazolyl chalcones. The role of electron withdrawing group in improving the anti-bacterial activity is supported by the findings of Sharma et al. ${ }^{22}$

3. The presence of electron donating methoxy group at $p$-position of the phenyl nucleus attached to position-4 of pyrazole through enone moiety improved the anti-fungal activity of the pyrazolyl chalcones. The role of electron releasing groups in improving the anti-fungal activity is supported by our recent findings. ${ }^{12}$

4. The introduction of heterocyclic moiety furan doesn't improve the anti-microbial spectrum of pyrazolyl chalcones. The results are similar to one of our previous studies. ${ }^{23}$

5. From these result we may conclude that different structural modifications are required for a compound to be effective against anti-bacterial and anti-fungal targets. This is in accordance with the results of Sortino et l. $^{24}$

\section{Conclusions}

In summary, we have synthesized s-cis (E)-1-(5-hydroxy3-methyl-1-phenyl-1 $H$-pyrazol-4-yl)-3-arylprop-2-en1-ones (2) in good yield and then isomerized to s-trans (E)-4-(3-(phenyl/aryl/heteroaryl)acryloyl)-5methyl-2-phenyl-1 $H$-pyrazol-3(2H)-ones (3) using iodobenzene diacetate in dichloromethane at room temperature in excellent yield. The structures of these $\alpha, \beta$-unsaturated ketones (pyrazolyl ketones $\mathbf{2}$ and $\mathbf{3}$ ) were established with the help of NMR, 2D NMR and HRMS techniques. The cytotoxicity of pyrazolyl chalcones showed that $\mathbf{2 c}$ is active at very low concentrations $\mathrm{IC}_{50} 13.3 \mu \mathrm{M}$ against colon cancer cell line (HCT-116). These compounds were also tested in vitro for their anti-bacterial activity against gram-positive and gram-negative bacteria and as well as for anti-fungal activity. The compounds $\mathbf{2 d}$ and $\mathbf{3 d}$ showed significant anti-bacterial activity against E. coli and B. subtilis, respectively and $\mathbf{2} \mathbf{b}$ and $\mathbf{3 b}$ showed significant anti-fungal activity against $C$. albicans and $A$. niger respectively.

\section{Supplementary Information}

The supplementary data i.e., NMR, 2D NMR and graphs of cytotoxic activity, associated with this article are available at www.ias.ac.in/chemsci.

\section{Acknowledgements}

Mahavir Parshad thanks University Grants Commission, New Delhi for financial support and award of teacher fellowship to Mahavir Parshad under FIP scheme $12^{\text {th }}$ plan period. 


\section{References}

1. Sahu K N, Balbhadra S S, Choudhary J and Kohli V D 2012 Curr. Med. Chem. 19209

2. Katsori A M and Hadjipavlou-Litina D 2009 Curr. Med. Chem. 161062

3. Go M L, Wu X and Liu X L 2005 Curr. Med. Chem. 12 481

4. Batovska D I and Todorova I T 2010 Curr. Clin. Pharmacol. 51

5. Siddiqui Z N, Mohammed T N, Ahmad A and Khan A U 2011 Bioorg. Med. Chem. Lett. 212860

6. Shelke S N, Mhaske G R, Bonifacio V D B and Gawande M B 2012 Bioorg. Med. Chem. Lett. 225727

7. Nagaraju M, Deepthi E G, Ashwini C, Vishnuvardhan M V P S, Nayak V L, Chandra R, Ramakrishna S and Gawali B B 2012 Bioorg. Med. Chem. Lett. 224314

8. Roman B I, De Ryck T, Dierickx L, Vanhoecke B W A, Katritzky A R, Bracke M and Stevens C V 2012 Bioorg. Med. Chem. Lett. 204812

9. Bandgar B P, Gawande S S, Bodade R G, Gawande N M and Khobragade C N 2009 Bioorg. Med. Chem. Lett. 17 8168

10. Insuasty B, Tigreros A, Orozco F, Quiroga J, Abonia R, Nogueras M, Sanchez A and Cobo J 2010 Bioorg. Med. Chem. 184965

11. (a) Moriarty R M and Prakash O 2008 In Hypervalent Iodine in Organic Chemistry: Chemical Transformations (UK: Wiley-Blackwell); (b) Wirth T 2003 Top. Curr. Chem. 224 1; (c) Zhdankin V V and Stang P J 2008 Chem. Rev. 108 5299; (d) Varvoglis A 2009 ARKIVOC 1

12. Verma V, Singh K, Kumar D, Klapotke T M, Stierstorfer J, Narasimhan B, Qazi A K, Hamid A and Jaglan S 2012 Eur. J. Med. Chem. 56195
13. Mohanty S K, Sridhar R, Padmanavan S Y, Sundar R and Mittra A S 1977 Indian J. Chem. 15B 1146

14. Houghton P, Fang R, Techatanawat I, Steventon G, Hylands P J and Lee C C 2007 Methods 42377

15. Marchetti F, Pettinari C and Pettinari R 2005 Coordination Chem. Rev. 2492909

16. Jensen B S 1959 Acta Chem. Scand. 131668

17. Espinoza-Hicks J C, Rodriguez-Valdez L M, NevarezMoorillon G V and Camacho-Davila A $2012 \mathrm{~J}$. Mol. Struct. 102088

18. (a) Garcia J I, Mayorala J A, Salvatell L, Assfeld X and Ruiz-Lopez M F 1996 J. Mol. Struct. (Theochem) 362 187; (b) Thirunarayanan G, Gopalakrishnan M and Vanangamudi G 2007 Spectrochim. Acta Part A 67 1106; (c) Yong Y, Ahn S, Hwang D, Yoon H, Jo G, Kim Y H, Kim S H, Kohb D and Lima Y 2013 Magn. Reson. Chem. 51364

19. (a) Kumar D, Singh S P, Martinez A, Fruchier A, Elguero J, Martinez-Ripoll M, Carrio J S and Virgilli A 1995 Tetrahedron 51 4891; (b) Holzer W, Kautsch C, Laggner C, Claramunt R M, Perez-Torralba M, Alkorta I and Elguero J 2004 Tetrahedron 606791

20. Pharmacopoeia of India 1996 Ministry of Health Department, Govt. of India (New Delhi) II p A-88

21. Cappucino J G and Sherman N 1999 In Microbiology-A Laboratory Manual (California: Addison Wesley) p 263

22. Sharma P, Rane N and Gurram V K 2004 Bioorg. Med. Chem. Lett. 144185

23. Kumar P, Narasimhan B, Sharma D, Judge V and Narang R 2009 Eur. J. Med. Chem. 441853

24. Sortino M, Delgado P, Jaurez S, Quiroga J, Abonia R, Insuasty B, Nogueras M, Rodero L, Garibotto F M, Enriz R D and Susana S A 2007 Bioorg. Med. Chem. 15 484 